\title{
The effect of apixaban on the pharmacokinetics of digoxin and atenolol in healthy subjects
}

This article was published in the following Dove Press journal:

Clinical Pharmacology:Advances and Applications

23 February 2017

Number of times this article has been viewed

\section{Charles Frost ${ }^{1}$ \\ Yan Song' \\ Zhigang $\mathrm{Yu}^{2}$ \\ Jessie Wang ${ }^{3}$ \\ Lois S Lee ${ }^{4}$ \\ Alan Schuster ${ }^{5}$ \\ Allyson Pollack' \\ Frank LaCreta'}

'Exploratory Clinical and Translational Research, Bristol-Myers Squibb, Princeton, NJ, USA; ${ }^{2}$ Medical Sciences, Amgen Asia R\&D Center, Shanghai, People's Republic of China; ${ }^{3}$ Global Biometric Sciences, Bristol-Myers Squibb, Princeton, NJ, ${ }^{4}$ Clinical Research, Intercept Pharmaceuticals, San Diego, CA, ${ }^{5}$ Bioanalytical Sciences, Bristol-Myers Squibb, Princeton, NJ, USA
Correspondence: Yan Song Exploratory Clinical and Translational Research, 8AI.18, Bristol-Myers Squibb, 3I I Pennington Rocky Hill Road, Pennington, NJ 08534-2130, USA

$\mathrm{Tel}+\mathrm{I} 6098183561$

$\mathrm{Fax}+\mathrm{I} 6098183220$

Email Yan.Song@bms.com
Purpose: Apixaban is often coadministered with treatments for cardiovascular comorbidities, which may lead to unintended drug-drug interactions (DDIs). The effects of apixaban on pharmacokinetics (PK) of multidose Lanoxin ${ }^{\circledR}$ (digoxin) and single-dose Tenormin ${ }^{\circledR}$ (atenolol) and the effects of single-dose atenolol on apixaban PK in healthy subjects were investigated in two Phase 1 studies.

Patients and methods: The digoxin DDI study was an open-label, multidose, two-treatment, single-sequence study in which subjects received digoxin $0.25 \mathrm{mg}$ q6h on day 1 , then once daily on days $2-10$, followed by apixaban $20 \mathrm{mg}$ and digoxin $0.25 \mathrm{mg}$ once daily on days $11-20$. The atenolol DDI study was an open-label, single-dose, randomized, three-period, three-treatment, crossover study in which subjects received a single oral dose of apixaban $10 \mathrm{mg}$, atenolol $100 \mathrm{mg}$, or apixaban $10 \mathrm{mg}$ plus atenolol $100 \mathrm{mg}$. The $90 \%$ confidence intervals (CIs) for the ratios of geometric means of peak plasma concentration $\left(\mathrm{C}_{\max }\right)$ and area under the concentration-time curve $\left(\mathrm{AUC}_{\mathrm{tau}}\right)$, with and without apixaban were calculated. Absence of effect was concluded if the point estimates and $90 \%$ CI were within the equivalence interval of $80 \%-125 \%$ (digoxin) or $70 \%-143 \%$ (atenolol). A similar analysis was performed to assess the effect of atenolol on apixaban.

Results: Apixaban had no clinically relevant effect on the PK of either atenolol or digoxin: point estimates and $90 \% \mathrm{CI}$ for both digoxin and atenolol $\mathrm{C}_{\max }$ and $\mathrm{AUC}$ were entirely within their respective no-effect intervals. Apixaban $\mathrm{C}_{\max }$ and $\mathrm{AUC}_{\text {inf }}$ were slightly decreased (ie, $18 \%$ and $15 \%$ lower, respectively) following atenolol coadministration. No serious or major bleedingrelated adverse events were reported during either study.

Conclusion: Apixaban had no effect on the PK of digoxin and there was no clinically relevant interaction between apixaban and atenolol. Coadministration of digoxin or atenolol with apixaban in healthy subjects was generally well tolerated.

Keywords: oral anticoagulant, factor Xa inhibitor, drug-drug interaction, Phase 1

\section{Introduction}

Apixaban, an oral direct factor Xa inhibitor, is indicated for the prevention of stroke and systemic embolism in nonvalvular atrial fibrillation (NVAF). ${ }^{1-4}$ Apixaban has also been approved for the prophylaxis of deep vein thrombosis (DVT) in patients who have undergone hip or knee replacement surgery, ${ }^{1,2,5-7}$ as well as for the treatment of DVT and pulmonary embolism (PE), and for the reduction of the risk of recurrent DVT and PE following initial therapy. ${ }^{1,2,8,9}$ Apixaban is often coadministered with treatments for cardiovascular comorbidities, such as hypertension, congestive heart failure, and diabetes. Therefore, it is important to assess the potential for pharmacokinetic interactions between apixaban and possible concomitant cardiovascular treatments. 
The pharmacokinetics (PK) of apixaban in healthy subjects are characterized by a time to peak plasma concentration $\left(\mathrm{T}_{\max }\right)$ of 3-4 hours and a mean elimination half-life $\left(\mathrm{t}_{1 / 2}\right)$ of $\sim 12$ hours. ${ }^{2,10}$ Apixaban is $87 \%$ bound to serum proteins, and is eliminated through multiple mechanisms, including cytochrome P450 3A4 (CYP3A4)-mediated metabolism, and renal, biliary, and direct intestinal excretion..$^{2,10-13}$ In addition to CYP3A4, apixaban is also a substrate of the drug efflux transporter, P-glycoprotein (P-gp)., ${ }^{2,10,14}$ Therefore, strong inhibitors and inducers of CYP3A4 and P-gp increase and decrease apixaban exposure, respectively. ${ }^{15,16}$ Apixaban does not inhibit or induce CYP3A4 or other CYP isoenzymes, and does not inhibit P-gp. ${ }^{14,17}$ Therefore, it is unlikely that apixaban would alter the metabolic clearance of concomitant medications that undergo CYP enzyme-mediated metabolism, and an interaction between apixaban and P-gp substrates is not expected.

Lanoxin $^{\circledR}$ (digoxin), a cardiac glycoside used for the treatment of congestive heart failure as well as atrial fibrillation, ${ }^{18,19}$ has a narrow therapeutic index. Because it is likely that apixaban and digoxin will be coadministered in some patients, it is important to evaluate the potential for apixaban to alter the PK of digoxin. Oral digoxin is rapidly absorbed, with a $\mathrm{T}_{\max }$ of 1-3 hours, and is eliminated slowly, with a mean $t_{1 / 2}$ of 1.5-2.0 days. ${ }^{19}$ Approximately $25 \%$ of digoxin in the plasma is protein-bound, and only $16 \%$ of the dose is metabolized, with $50 \%-70 \%$ excreted unchanged in the urine. ${ }^{19}$ Metabolism of digoxin is independent of CYP, and digoxin does not appear to induce or inhibit the CYP system. ${ }^{19}$ The potential for a drug-drug interaction (DDI) between apixaban and digoxin was considered to be low on the basis of their individual absorption, distribution, metabolism, and excretion profiles. Digoxin also serves as an acceptable P-gp probe substrate to predict the effects of P-gp modulators used in the clinical setting. ${ }^{20}$

Tenormin ${ }^{\circledR}$ (atenolol) is a cardioselective beta-blocker that is prescribed to treat hypertension. ${ }^{21}$ The PK of oral atenolol is characterized by a $\mathrm{T}_{\max }$ of $2-4$ hours, and a mean elimination $t_{1 / 2}$ of 6-7 hours. Atenolol minimally binds to plasma protein $(6 \%-16 \%)$. Half of the oral atenolol dose is excreted unchanged in the feces; most of the remainder is eliminated through renal excretion, with little or no metabolism by the liver. ${ }^{21}$ Beta-blockers may reduce hepatic blood flow ${ }^{22}$; however, apixaban clearance is not expected to be limited by reduced hepatic blood flow because it is not a high extraction ratio drug. ${ }^{2}$ Although atenolol is not metabolized by CYP enzymes and does not inhibit P-gp-mediated transport, both apixaban and atenolol are P-gp substrates. ${ }^{14,23}$
Two separate Phase 1 studies were conducted to evaluate the risk of clinically meaningful DDIs between apixaban and digoxin or atenolol. Based on the pharmacokinetic characteristics of these drugs, interactions between apixaban and digoxin and between apixaban and atenolol were expected to be minimal. The primary objective of the digoxin DDI study (NCT02262520) was to assess the effect of multidose apixaban on the PK of multidose digoxin in healthy subjects. The primary objectives of the atenolol DDI study (NCT02262533) were to assess the effect of atenolol on the PK of apixaban and the effect of apixaban on the PK of atenolol in healthy subjects.

\section{Materials and methods Study design}

The digoxin DDI study was an open-label, multidose, two-treatment, single-sequence study in healthy subjects (Figure 1A). The atenolol DDI study was an open-label, single-dose, randomized, three-period, three-treatment, crossover study in healthy subjects (Figure 1B). The protocols for both studies were approved by the New England Institutional Review Board (Wellesley, MA, USA) and the studies were conducted in accordance with Title 21, Part 56 Code of Federal Regulations (CFR) and Title 21, Part 50 CFR, the principles of the Declaration of Helsinki, Good Clinical Practice as defined by the International Conference on Harmonisation, and the ethical principles underlying the European Union directive 2001/20/EC.

\section{Subjects}

Both studies recruited male and female subjects aged 18-45 years with a body mass index of $18-30 \mathrm{~kg} / \mathrm{m}^{2}$ who were considered to be healthy according to medical history, physical examination, vital signs, 12-lead electrocardiogram (ECG) assessment, and clinical laboratory tests. Subjects underwent screening evaluations within a 21-day period prior to dosing to determine eligibility. Both trials excluded female subjects who were pregnant or nursing. The digoxin study excluded all women of childbearing potential; the atenolol DDI study allowed them to participate if they used accepted methods of contraception, excluding use of hormonal contraception, within 3 months of study start. In both studies, all subjects provided written informed consent prior to the initiation of any study-specific procedures.

Subjects were excluded from the digoxin DDI study if they had relevant arrhythmias, unexplained syncope, abnormal renal function, or conditions that can cause electrolyte disorder. Subjects were excluded from the atenolol DDI study if they had a history of asthma, bronchitis or other significant pulmonary disease, or a history of hypotension. For both 
A

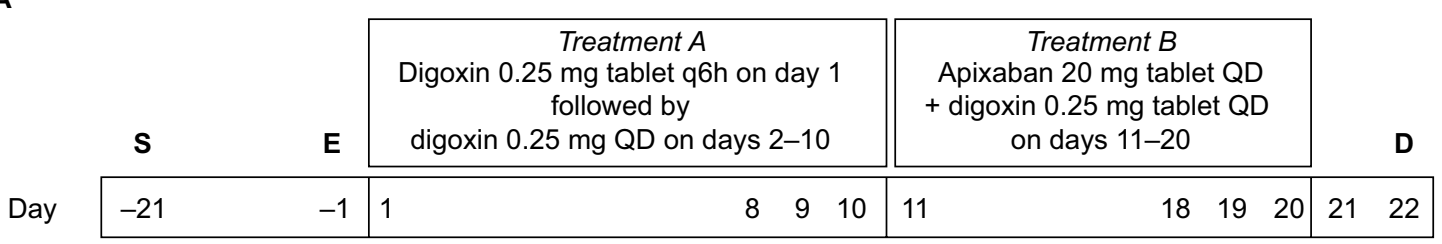

B

Period 1

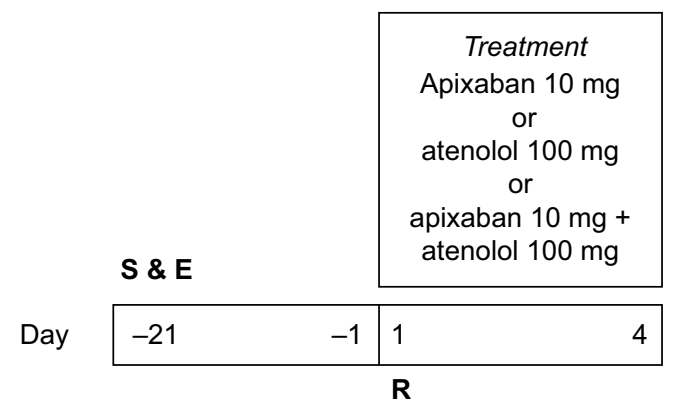

Period 2

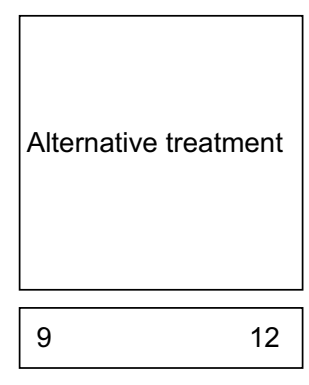

Period 3

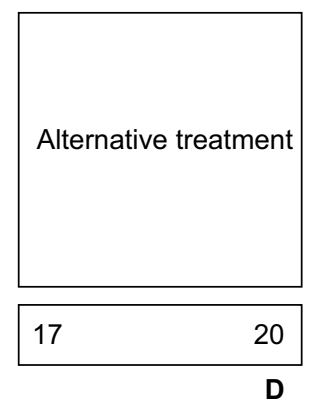

Figure I Study design: (A) apixaban-digoxin PK study; (B) apixaban-atenolol PK study.

Abbreviations: D, study discharge; E, enrollment; PK, pharmacokinetics; q6h, every 6 hours; QD, once daily, R, randomization; S, screening.

studies, exclusion criteria also included a history or evidence of abnormal bleeding or coagulation disorder and/or having a first-degree relative under 50 years of age with a history of abnormal bleeding or coagulation disorder, significant gastrointestinal (GI) disease (within 3 months) or GI surgery that could affect absorption of study drug, a history of significant drug allergies, or prior exposure to apixaban. Other exclusion criteria included exposure to any investigational drug within 4 weeks of study start, prescription drugs within 4 weeks of study start, or any over-the-counter medications or herbal preparations within 1 week (digoxin study) or 2 weeks (atenolol study) of study start.

\section{Treatment}

For both studies, subjects were admitted to the clinical facility on the day before study drug administration and remained there until clinical and laboratory safety data evaluations had been completed (day 22 for the digoxin DDI study and day 4 of period 3 for the atenolol DDI study).

In the digoxin DDI study, subjects were assigned to receive a digoxin $0.25 \mathrm{mg}$ tablet $\mathrm{q} 6 \mathrm{~h}$ on day 1 and a digoxin $0.25 \mathrm{mg}$ tablet once daily on days $2-10$, followed by an apixaban $20 \mathrm{mg}$ tablet once daily and a digoxin $0.25 \mathrm{mg}$ tablet once daily on days 11-20. The dose and dosing regimen for digoxin were selected to mimic a commonly administered digoxin dosing regimen in the clinical setting, and to achieve, but not exceed, the therapeutic concentration range for atrial fibrillation $(\sim 1.4-2.5 \mathrm{ng} / \mathrm{mL}){ }^{24,25}$ Apixaban $20 \mathrm{mg}$ once daily was selected to represent the highest dose evaluated in Phase 2 clinical trials at the time the study was conducted. ${ }^{26-28}$ Digoxin $0.25 \mathrm{mg}$ tablets were provided by the investigator and manufactured by GlaxoSmithKline (Research Triangle Park, NC, USA), and apixaban $20 \mathrm{mg}$ tablets were supplied and manufactured by Bristol-Myers Squibb Company (New Brunswick, NJ, USA).

Subjects in the atenolol DDI study were randomly assigned to receive a single oral dose of apixaban $10 \mathrm{mg}$, atenolol $100 \mathrm{mg}$, or apixaban $10 \mathrm{mg}+$ atenolol $100 \mathrm{mg}$ on day 1 of period 1 . The alternative treatments were administered during periods 2 and 3 according to a randomization schedule. Treatment periods were separated by a 4-day washout interval. An atenolol dose of $100 \mathrm{mg}$ was chosen for this study as representative of the upper end of the dose range. ${ }^{21,29}$ An apixaban dose of $10 \mathrm{mg}$ was selected on the basis of safety data available at the time, and reflected exposures likely to be observed in patients, as the Phase 3 clinical trials evaluated apixaban doses ranging from 2.5 to $10 \mathrm{mg}$ twice daily for reducing the risk of stroke in NVAF, prophylaxis of thrombosis-related events following hip or knee replacement surgery, or treatment of DVT and PE. ${ }^{3-9,30}$ Atenolol 100-mg tablets were supplied by the investigator and manufactured by AstraZeneca Pharmaceuticals LP (Wilmington, DE, USA), and apixaban 5-mg tablets were supplied and manufactured by Bristol-Myers Squibb Company. 


\section{Sample collection}

In both studies, blood samples for analysis were collected via indwelling catheter or by direct venipuncture. In the digoxin DDI study, blood samples for digoxin PK were collected at selected times over the 24-hour dosing interval on days 10 and 20. Blood samples for digoxin trough concentrations were collected predose on days $8,9,10,18,19$, and 20. Blood samples for apixaban trough concentrations were collected predose on days 18-20. For apixaban and digoxin pharmacokinetic analysis, each $5 \mathrm{~mL}$ blood sample was gently inverted a few times immediately after collection to ensure complete mixing (with anticoagulant [sodium citrate] or the clot activator gel for apixaban and digoxin, respectively) in the serum-separating tube and was then placed on chipped ice. Samples for pharmacokinetic analysis of apixaban (within 15 minutes) and digoxin (after a 30- to 60-minute wait to allow for clotting) were centrifuged for 15 minutes at $\sim 1500 \times g$ at room temperature to separate the plasma and serum, respectively. Separated plasma (apixaban) and serum (digoxin) were transferred to cryogenic vials, immediately stored at or below $-20^{\circ} \mathrm{C}$, and sent for analysis (apixaban: Intertek Pharmaceutical Services, El Dorado, CA, USA; digoxin: Advion Biosciences, Ithaca, NY, USA).

For the atenolol DDI study, blood samples for PK assessment were collected both predose and over a period of 72 hours postdose for apixaban and through 48 hours postdose for atenolol. For atenolol and apixaban PK analyses, each 3.0 and $2.7 \mathrm{~mL}$ blood sample, respectively, was gently inverted a few times immediately after collection to ensure complete mixing with anticoagulant $\left(\mathrm{K}_{2}\right.$ EDTA for atenolol, $3.2 \%$ sodium citrate for apixaban) and then placed on chipped ice. Within 15 minutes, each blood sample was centrifuged for 15 minutes at $\sim 1500 \times g$ at $4{ }^{\circ} \mathrm{C}$ to separate the plasma, and was then transferred to a cryogenic vial, immediately stored at or below $-20^{\circ} \mathrm{C}$, and sent for analysis (apixaban: Intertek Pharmaceutical Services; atenolol: Vimta Labs, Andhra Pradesh, India).

\section{Pharmacokinetic analysis}

In both studies, the serum (digoxin) and plasma (atenolol and apixaban) samples were analyzed using validated liquid chromatography mass spectrometry/mass spectrometry (LC/MS/MS) methods. ${ }^{31}$ The between-run and within-run variabilities for digoxin in serum quality-control samples were $\leq 4.6 \%$ and $\leq 8.0 \%$, respectively, with deviations from nominal concentration of within $\pm 3.3 \%$. Digitoxin was used as the internal standard. The between-run and within-run variabilities for atenolol in plasma quality-control samples were $\leq 6.3 \%$ and $\leq 8.6 \%$, respectively, with deviations from nominal concentration of within $\pm 8.4 \%$ (data on file, BMS, 2008). Atenolol- $\mathrm{d}_{7}$ was used as the internal standard. The lower limit of quantification is 0.1 and $1.0 \mathrm{ng} / \mathrm{mL}$ for digoxin and atenolol, respectively. All samples were analyzed within the period of analyte stability. Individual subject pharmacokinetic parameter values were derived by standard noncompartmental methods using Kinetica ${ }^{\circledR}$ within the eToolbox ${ }^{\circledR}$ software package (Thermo Fisher Scientific, Waltham, MA, USA).

The digoxin DDI study assessed the following digoxin parameters: peak plasma concentration $\left(\mathrm{C}_{\max }\right)$, trough observed concentration $\left(\mathrm{C}_{\min }\right), \mathrm{T}_{\max }$, and the area under the plasma concentration-time curve over one dosing interval $\left(\mathrm{AUC}_{\mathrm{tau}}\right)$ for digoxin on days 10 and 20. In addition, $\mathrm{C}_{\min }$ for apixaban was assessed on days 18-20.

In the atenolol DDI study, single-dose pharmacokinetic parameters assessed for apixaban and atenolol included $\mathrm{C}_{\max }$, $\mathrm{T}_{\max }$, the AUC from time 0 to the time of last quantifiable concentration following a single dose $\left(\mathrm{AUC}_{0-t}\right)$, the AUC from time 0 extrapolated to infinite time following a single dose $\left(\mathrm{AUC}_{\mathrm{inf}}\right)$, and the plasma $\mathrm{t}_{1 / 2}$.

\section{Safety}

Subjects were closely monitored throughout both studies for adverse events (AEs), and were not discharged from the study until the investigator determined that all AEs had resolved or were not of clinical significance. Data on AEs were collected through constant monitoring, volunteering of information by the study participants, and daily questioning by the medical staff. Additionally, AEs could be identified by investigator review of vital signs, ECGs, and laboratory and other data. All AEs were coded and grouped by system organ class, preferred term, treatment, and severity according to the Medical Dictionary for Regulatory Activities criteria (version 8.1 for digoxin study and 10.0 for atenolol study).

\section{Statistical analysis}

In the digoxin DDI study, all available data from subjects who received digoxin were included in the digoxin PK data set, but only subjects with sufficient data points to establish a complete pharmacokinetic profile from both treatments were included in the summary statistics and statistical analysis. The apixaban data set included all available data from subjects who received apixaban.

In the atenolol DDI study, all available concentrationtime data from subjects who received any apixaban or atenolol were reported. All available derived pharmacokinetic parameter values were included in the PK data set and 
reported, but only subjects with an evaluable pharmacokinetic profile were included in the summary statistics and statistical analysis.

In both studies, all available data for subjects who received study medication were included in the safety data sets. All statistical analyses were carried out using SAS/ STAT $^{\circledR}$ version 8.2 (SAS Institute, Cary, NC, USA).

In the digoxin DDI study, summary statistics were tabulated for the digoxin pharmacokinetic parameters by treatment. $\mathrm{C}_{\min }$ for apixaban and digoxin were summarized by study day. A linear mixed-effect model with treatment as fixed effect and subject as repeated measures was fitted to the log-transformed pharmacokinetic parameters $\left(\mathrm{C}_{\max }\right.$, AUC) for use in estimation of effects and construction of confidence intervals (CIs). No adjustments were made for multiplicity. An absence of an effect of apixaban on digoxin pharmacokinetic parameters would be concluded if the $90 \%$ $\mathrm{CIs}$ for the ratios of the geometric means for digoxin $\mathrm{C}_{\text {max }}$ and $\mathrm{AUC}_{\text {tau }}$, with and without apixaban, were within the equivalence interval of $80 \%-125 \%$.

If apixaban had no effect on the PK of digoxin, then data from 20 subjects would have provided $96 \%$ power to conclude absence of effect with respect to $\mathrm{C}_{\max }$ and $94 \%$ power to conclude absence of effect with respect to $\mathrm{AUC}_{\text {tau. }}$. These calculations used the approach described by Diletti et $\mathrm{al}^{32}$ and assumed log-normal distributions of $\mathrm{C}_{\max }$ and $\mathrm{AUC}_{0-t}$ for digoxin with intrasubject coefficients of variation (CVs) of $18 \%$ and $20 \%$, respectively, as reported by Mant et al. ${ }^{33}$ Twenty-four subjects were enrolled to allow for possible dropouts.

In the atenolol DDI study, summary statistics were tabulated for apixaban and atenolol pharmacokinetic parameters by treatment. A linear mixed-effect model with treatment, period, and sequence as fixed effects and subject as repeated measures was fitted to the log-transformed pharmacokinetic parameters $\left(\mathrm{C}_{\max }\right.$ and $\left.\mathrm{AUC}\right)$ for use in estimation of effects and construction of CIs. No adjustments were made for multiplicity. An absence of effect of one drug on the PK of the other drug would be concluded if the $90 \%$ CIs for the ratios of geometric means for $\mathrm{C}_{\text {max }}$ and $\mathrm{AUC}_{\text {inf }}$ with and without the coadministered drug were within the clinically meaningful equivalence interval of $70 \%-143 \%$. This CI was selected because atenolol is a cardioselective beta-blocker that has limited metabolic drug interaction potential, is not a narrow therapeutic index drug, and is dosed to pharmacological effect. ${ }^{21}$ In addition, there is sufficiently understood clinical relevance of changes in exposure to apixaban. This approach has been used with other drugs without DDI liabilities. ${ }^{34,35}$ If atenolol $100 \mathrm{mg}$ had no effect on the PK of apixaban, 12 subjects would have provided $94 \%$ power to conclude absence of effect with respect to $\mathrm{C}_{\max }$ and $99 \%$ power to conclude absence of effect with respect to $\mathrm{AUC}_{\text {inf. }}$. These calculations used the approach described by Diletti et $\mathrm{al}^{32}$ and assumed $\mathrm{C}_{\max }$ and $\mathrm{AUC}_{\text {inf }}$ were log-normally distributed with intrasubject standard deviations (SDs) of 0.23 for $\log \left(\mathrm{C}_{\max }\right)$ and 0.16 for $\log \left(\mathrm{AUC}_{\mathrm{inf}}\right)$ for apixaban, as derived from data reported by Yamahira et al. ${ }^{30}$ If apixaban $10 \mathrm{mg}$ had no effect on the PK of atenolol, 12 subjects would have provided $99 \%$ power to conclude absence of effect with respect to both $\mathrm{C}_{\max }$ and $\mathrm{AUC}_{\text {inf. }}$. These calculations assumed $\mathrm{C}_{\max }$ and $\mathrm{AUC}_{\mathrm{inf}}$ were log-normally distributed with intrasubject $\mathrm{CV}$ of $19 \%$ for $\mathrm{C}_{\max }$ and $13 \%$ for $\mathrm{AUC}_{\text {inf }}$ for atenolol, as derived from Czendlik et al. ${ }^{36}$ Fifteen subjects were enrolled to allow for possible dropouts, with no replacements allowed for discontinuations.

\section{Results}

\section{Subjects}

In the digoxin DDI study, a total of 24 healthy male subjects were enrolled and received treatment A (digoxin $0.25 \mathrm{mg}$ ), and 23 of those subjects also received treatment B (digoxin $0.25 \mathrm{mg}+$ apixaban $20 \mathrm{mg}$ ). One subject discontinued after day 8 of treatment A because of an AE of elevated alanine transaminase (ALT) and another received study drug through day 19 and withdrew consent on day 20. There were no protocol deviations that would impact study results. An overview of subject demographics and baseline characteristics is shown in Table 1.

A total of 15 healthy male and female subjects were randomized and received treatment in the atenolol DDI study. Of the 15 treated subjects, 14 completed the study and one discontinued after receiving apixaban alone and apixaban plus atenolol, owing to an AE (cellulitis, considered unrelated to study medication). There were no protocol deviations that affected the trial outcomes or conclusions. An overview of subject demographics and baseline characteristics is shown in Table 1 .

\section{PK}

The mean concentration versus time profiles of digoxin were similar when administered alone or with apixaban (Figure 2) and the $90 \%$ CIs for the ratios of geometric means of digoxin $\mathrm{C}_{\max }$ and $\mathrm{AUC}_{\text {tau }}$ (versus without apixaban) were within the prespecified equivalence criteria of $80 \%-125 \%$ (Table 2), indicating the absence of an interaction. The mean SD daily $\mathrm{C}_{\text {min }}$ values of digoxin were similar during treatment with 
Table I Baseline characteristics and demographics

\begin{tabular}{lll}
\hline Characteristic & $\begin{array}{l}\text { Apixaban-digoxin } \\
\text { PK study (N=24) }\end{array}$ & $\begin{array}{l}\text { Apixaban-atenolol } \\
\text { PK study (N=15) }\end{array}$ \\
\hline $\begin{array}{ll}\text { Age, years } \\
\text { Mean }\end{array}$ & 31 & 29 \\
SD & 7 & 8 \\
Range & $22-45$ & $18-44$ \\
Sex, n (\%) & & \\
Male & $24(100)$ & $13(87)$ \\
Female & 0 & $2(13)$ \\
Race, n (\%) & & \\
White & $10(42)$ & $8(53)$ \\
Black & $11(46)$ & $7(47)$ \\
Asian & $3(13)$ & 0 \\
Ethnicity & & \\
Not Hispanic/Latino & $20(83)$ & $8(53)$ \\
Hispanic/Latino & $4(17)$ & $7(47)$ \\
Weight, kg & & \\
Mean & 73.8 & 80.9 \\
SD & 10.0 & 10.4 \\
Range & $51.0-91.4$ & $58.3-97.1$ \\
BMI, kg/m & & 26.4 \\
Mean & 24.3 & 1.9 \\
SD & 2.6 & $22.9-30.0$ \\
Range & $18.8-29.8$ & \\
\hline Abreviations: & &
\end{tabular}

Abbreviations: BMI, body mass index; PK, pharmacokinetics; SD, standard deviation.

digoxin $0.25 \mathrm{mg}$ (ranging from $0.52[0.20]$ to $0.57 \mathrm{ng} / \mathrm{mL}$ [0.19]) and digoxin $0.25 \mathrm{mg}$ plus apixaban $20 \mathrm{mg}$ (ranging from 0.46 [0.17] to $0.53 \mathrm{ng} / \mathrm{mL}$ [0.20]), indicating that digoxin was at steady state in both treatment periods. The mean (SD) $\mathrm{C}_{\min }$ values of apixaban on days 18,19 , and 20 were similar (ranging from 49.0 [19.5] to $49.6 \mathrm{ng} / \mathrm{mL}$ [23.3]), demonstrating that apixaban was at steady state during the PK assessment.
Table 2 Summary statistics for digoxin pharmacokinetic parameters

\begin{tabular}{|c|c|c|c|c|}
\hline \multirow[t]{2}{*}{ Parameter } & \multicolumn{2}{|l|}{ Digoxin } & \multicolumn{2}{|c|}{$\begin{array}{l}\text { Ratio of adjusted } \\
\text { geometric means }\end{array}$} \\
\hline & $\begin{array}{l}\text { Treatment A } \\
(n=22)\end{array}$ & $\begin{array}{l}\text { Treatment B } \\
(n=22)\end{array}$ & $\begin{array}{l}\text { Point } \\
\text { estimate, } \\
\text { B vs A }\end{array}$ & $90 \% \mathrm{Cl}$ \\
\hline $\begin{array}{l}\mathrm{C}_{\max } \\
(\mathrm{ng} / \mathrm{mL})\end{array}$ & $1.68(27)$ & $1.54(25)$ & 0.92 & $0.82,1.03$ \\
\hline $\begin{array}{l}\mathrm{AUC}_{\mathrm{tau}} \\
(\mathrm{ng} \cdot \mathrm{h} / \mathrm{mL})\end{array}$ & $16.8(28)$ & I5.I (28) & 0.90 & $0.84,0.96$ \\
\hline \multicolumn{5}{|l|}{$\mathrm{T}_{\max }(\mathrm{h})$} \\
\hline $\begin{array}{l}\text { Median } \\
\text { (min, max) }\end{array}$ & $1.00(0.5,2.0)$ & $1.00(1.0,2.0)$ & & \\
\hline
\end{tabular}

Notes: ${ }^{2}$ The ratio of geometric means is estimated from the linear mixed-effect model, with treatment as fixed effect and subject as repeated measure. Geometric mean ( $\mathrm{CV} \%$ ), unless otherwise noted. A, digoxin $0.25 \mathrm{mg}$, pharmacokinetic assessment on day 10. B, digoxin $0.25 \mathrm{mg}+$ apixaban $20 \mathrm{mg}$, pharmacokinetic assessment on day 20.

Abbreviations: $A \cup C_{t a u}$, area under the plasma concentration curve in one dosing interval; $\mathrm{C}_{\text {max }}$, maximum observed plasma concentration; $\mathrm{CV}$, coefficient of variation; min, minimum; max, maximum; $T_{\max }$, time of the maximum observed plasma concentration.

The mean concentration-time profiles of apixaban administered alone and with atenolol are shown in Figure 3A and summary statistics for apixaban pharmacokinetic parameters in the atenolol DDI study are shown in Table 3. Apixaban $\mathrm{C}_{\max }, \mathrm{AUC}_{0-\uparrow}$, and $\mathrm{AUC}_{\mathrm{inf}}$ values decreased by $18 \%, 15 \%$, and $15 \%$, respectively, when apixaban was coadministered with atenolol. Apixaban $\mathrm{T}_{\max }$ and $\mathrm{t}_{1 / 2}$ values were similar following administration alone and with atenolol (Table 3). The 90\% CIs for the ratios of geometric means of apixaban (versus without atenolol) were within the prespecified equivalence criteria of $70 \%-143 \%$. The mean concentration versus time

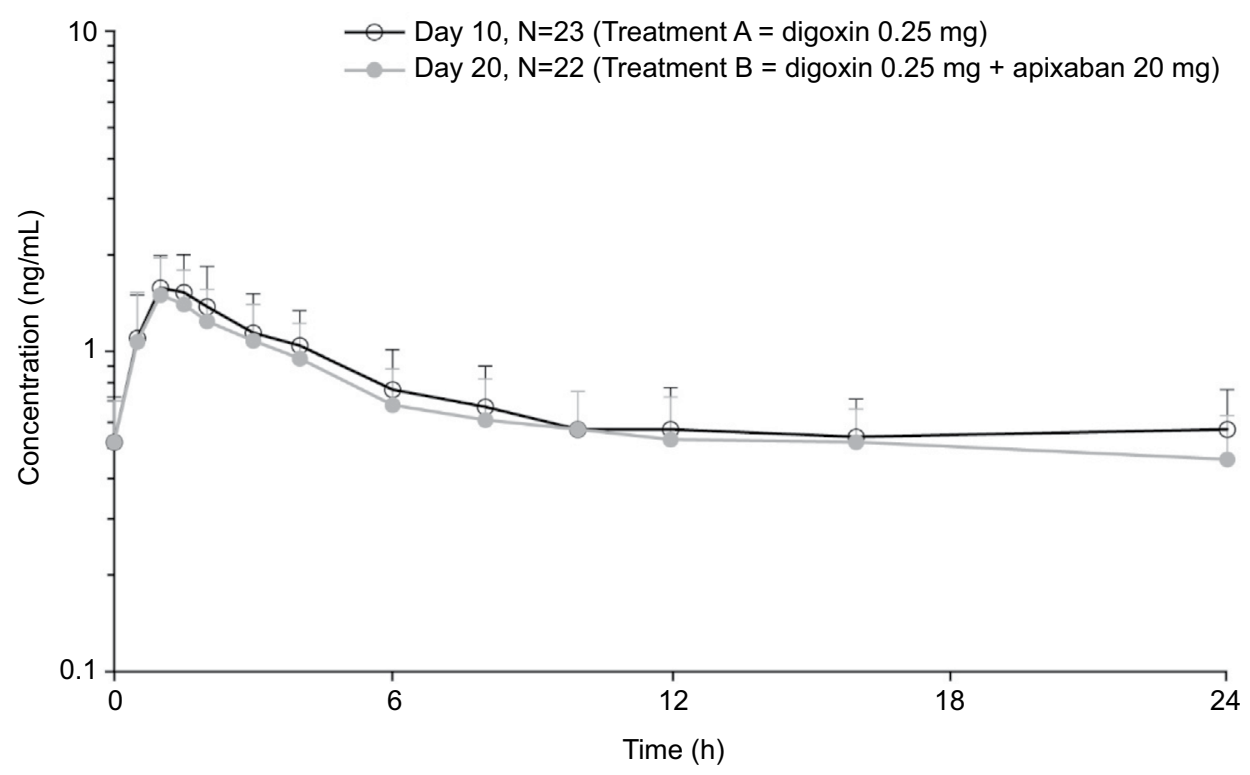

Figure 2 Mean serum digoxin concentrations versus time profiles when administered alone or with apixaban. Note: Error bars show $+\mathrm{I}$ standard deviation from the mean. 

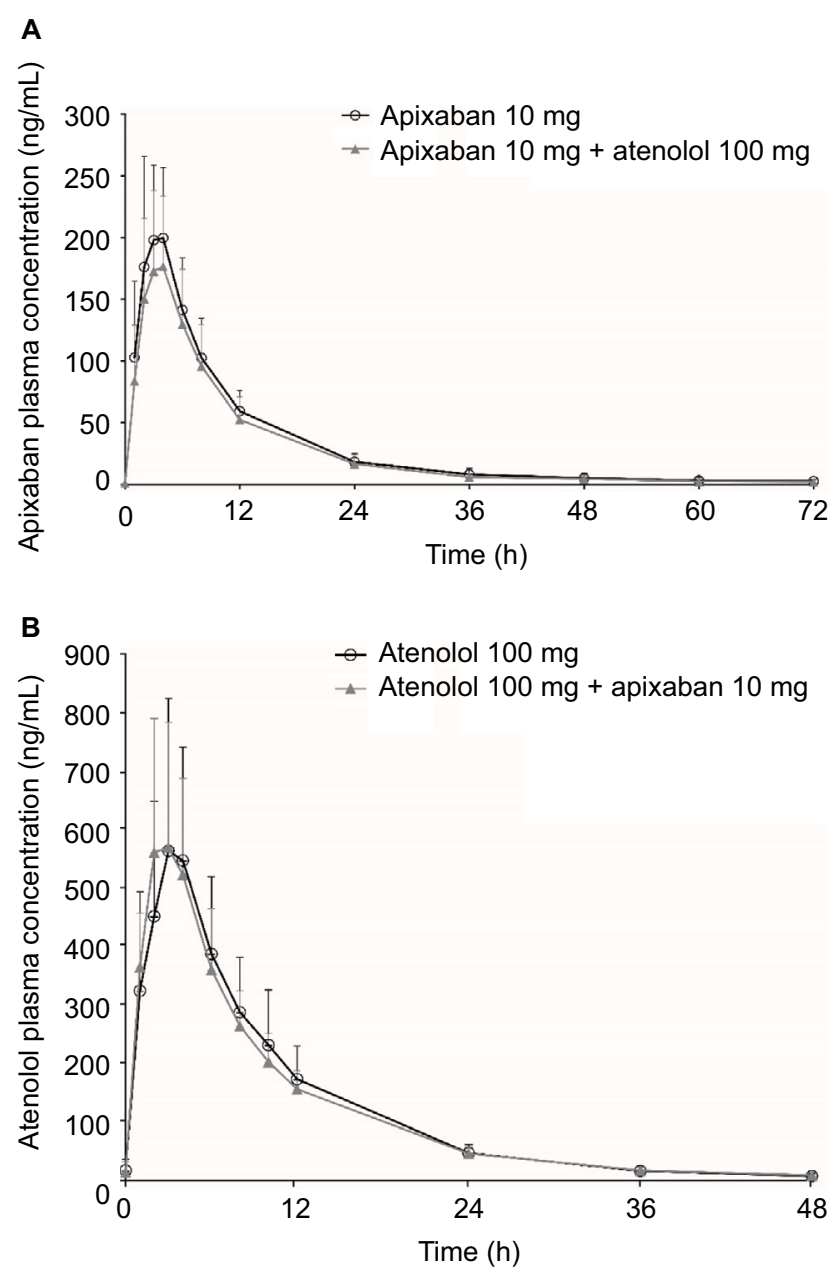

Figure 3 Mean plasma concentration versus time by treatment: (A) apixaban, (B) atenolol.

Note: Error bars show $+\mathrm{I}$ standard deviation from the mean.

profiles of atenolol administered alone and with apixaban are shown in Figure 3B and summary statistics for atenolol pharmacokinetic parameters are also shown in Table 3. No effect of apixaban on atenolol PK was observed, and the $90 \%$
CIs for the ratios of geometric means of atenolol (versus without apixaban) were within the prespecified equivalence criteria of $70 \%-143 \%$.

\section{Safety}

No deaths, serious AEs, or major bleeding-related AEs were reported during either study. All AEs reported in the digoxin DDI study were mild to moderate in intensity and resolved without treatment. The most frequently reported treatmentemergent AEs were an increase in ALT and ocular or scleral hyperemia, each reported in three subjects, and dizziness and pharyngolaryngeal pain, each reported in two subjects. Elevated ALT occurred during digoxin treatment and prior to the administration of apixaban in two subjects, one of whom discontinued the study before the start of apixaban treatment. A single bleeding-related AE of gingival bleeding occurred in one subject on day 18 , and resolved after 4 days without requiring interruption of study drug. The event was considered to be mild in intensity and possibly related to study drug by the investigator. Bruising around the intravenous catheter site occurred in one subject on day 12. This event was considered to be mild in intensity and was considered unlikely to be related to study medication. The subject completed the study without interruption of study therapy. No significant vital sign or ECG findings were reported.

Few AEs (five for apixaban and four for apixaban + atenolol) occurred during the atenolol DDI study, none of which were bleeding-related, with no appreciable difference between treatments. The most common AE was headache (three subjects). One subject discontinued due to moderate cellulitis that began on day 4 after administration of a single dose of apixaban + atenolol; this AE was considered unrelated

Table 3 Summary statistics for apixaban and atenolol pharmacokinetic parameters

\begin{tabular}{|c|c|c|c|c|c|c|c|c|}
\hline \multirow[t]{3}{*}{ Parameter } & \multicolumn{4}{|l|}{ Apixaban } & \multicolumn{4}{|l|}{ Atenolol } \\
\hline & \multirow[t]{2}{*}{$\begin{array}{l}\text { Treatment A } \\
(n=I 5)\end{array}$} & \multirow[t]{2}{*}{$\begin{array}{l}\text { Treatment C } \\
(n=14)\end{array}$} & \multicolumn{2}{|c|}{$\begin{array}{l}\text { Ratio of adjusted } \\
\text { geometric means }^{\mathrm{a}}\end{array}$} & \multirow[t]{2}{*}{$\begin{array}{l}\text { Treatment B } \\
(n=14)\end{array}$} & \multirow[t]{2}{*}{$\begin{array}{l}\text { Treatment C } \\
(n=\mid 5)\end{array}$} & \multicolumn{2}{|c|}{$\begin{array}{l}\text { Ratio of adjusted } \\
\text { geometric means }^{\mathrm{a}}\end{array}$} \\
\hline & & & $\begin{array}{l}\text { Point } \\
\text { estimate, } \\
\text { C vs A }\end{array}$ & $90 \% \mathrm{Cl}$ & & & $\begin{array}{l}\text { Point } \\
\text { estimate, } \\
\text { C vs B }\end{array}$ & $90 \% \mathrm{Cl}$ \\
\hline $\mathrm{C}_{\max }(\mathrm{ng} / \mathrm{mL})$ & $216(33)$ & $180(32)$ & 0.82 & $0.75,0.89$ & $603(39)$ & $598(33)$ & 0.98 & $0.84,1.13$ \\
\hline$A \cup C_{0-t}(\mathrm{ng} \cdot \mathrm{h} / \mathrm{mL})$ & $2103(3 \mathrm{I})$ & $1838(33)$ & 0.85 & $0.79,0.92$ & $5500(29)$ & $5528(22)$ & 1.00 & $0.90,1.11$ \\
\hline$A \cup C_{\text {inf }}(\mathrm{ng} \cdot \mathrm{h} / \mathrm{mL})$ & $2157(31)$ & $1878(33)$ & 0.85 & $0.78,0.92$ & 5596 (29) & $5649(22)$ & 1.00 & $0.91,1.11$ \\
\hline $\mathrm{t}_{1 / 2}(\mathrm{~h})$, mean $(\mathrm{SD})$ & $13.9(6.1)$ & 11.7 (3.8) & - & - & $8.33(1.64)$ & $8.57(1.89)$ & - & - \\
\hline $\begin{array}{l}\mathrm{T}_{\max }(\mathrm{h}), \text { median } \\
(\min , \max )\end{array}$ & $3(I, 4)$ & $4(2,4)$ & - & - & $3(1,6)$ & $2(2,4)$ & - & - \\
\hline
\end{tabular}

Notes: a The ratio of geometric means is estimated from the linear mixed-effect model, with treatment as fixed effect and subject as repeated measure. Geometric mean (CV \%), unless otherwise noted. A, apixaban $10 \mathrm{mg}$. B, atenolol $100 \mathrm{mg}$. C, apixaban $10 \mathrm{mg}+$ atenolol $100 \mathrm{mg}$.

Abbreviations: $A \cup C_{0-t}$, area under the plasma concentration curve from time 0 to time of the last quantifiable concentration following a single dose; AUC $C_{\text {inf }}$ area under the plasma concentration curve from time 0 extrapolated to time infinity; $\mathrm{Cl}$, confidence interval; $\mathrm{C}_{\max }$, maximum observed plasma concentration; $\mathrm{CV}$, coefficient of variation; min, minimum; max, maximum; $S D$, standard deviation; $\mathrm{t}_{1 / 2}$, plasma half-life; $\mathrm{T}_{\max }$, time of the maximum observed plasma concentration. 
to study treatment. No significant laboratory, vital sign, or ECG findings were reported.

\section{Discussion}

The two DDI studies reported here investigated potential pharmacokinetic interactions between apixaban and digoxin and apixaban and atenolol in healthy subjects. The results of both studies confirmed the hypothesis of little potential for a DDI between apixaban and either digoxin or atenolol. ${ }^{14,17,19,20}$ These studies are in agreement with previous studies indicating a low potential for apixaban to interact with concomitant medications. $^{12,14}$

In the digoxin DDI study, administration of multidose apixaban $20 \mathrm{mg}$ once daily did not affect the PK of multidose digoxin. With a multidose regimen, the digoxin DDI study was designed to evaluate the potential for a DDI at steadystate levels. Both apixaban and digoxin reached steady state (based on $\mathrm{C}_{\min }$ values) by the time of the individual pharmacokinetic evaluations. The achievement of apixaban steady state is consistent with previous observations. ${ }^{10}$ The $90 \%$ CIs for digoxin $\mathrm{C}_{\max }$ and $\mathrm{AUC}_{\text {tau }}$ geometric mean ratios were within the equivalence interval of $80 \%-125 \%$, indicating absence of an effect of apixaban on digoxin PK. Apixaban did not appear to affect the GI absorption of digoxin. The $\mathrm{T}_{\text {max }}$ of digoxin was unchanged following coadministration of apixaban. The findings provide support for the low potential for apixaban to affect the PK of other $\mathrm{P}$-gp substrates, as digoxin is a commonly used P-gp probe substrate. ${ }^{20}$ It should be noted that the apixaban dose in this study $(20 \mathrm{mg})$ is expected to result in steady-state exposure (i.e., $\mathrm{AUC}_{\text {tau }}$ ) that is $\sim 1.5-2$ times higher than that achieved in most patients treated with the highest approved apixaban dose, $10 \mathrm{mg}$ twice daily, for the treatment of venous thromboembolism (VTE), which provides further assurance that apixaban-mediated interactions with other P-gp substrates (eg, quinidine) are unlikely.

There was no impact of apixaban on the PK of atenolol observed in this study. The atenolol $\mathrm{T}_{\max }$ was slightly shortened from 3 to 2 hours; however, the point estimates for atenolol $\mathrm{C}_{\max }$ and $\mathrm{AUC}$ ratios with and without apixaban coadministration are close to $1(0.98-1.00)$, with the $90 \% \mathrm{CI}$ for $\mathrm{C}_{\max }$ and $\mathrm{AUC}_{\mathrm{inf}}$ ratios within the prespecified no-effect interval of $70 \%-143 \%$. It is even more important to note that the CIs observed for all of the exposure parameter point estimates fell between 0.84 and 1.13 , and would have also met the usual and narrower typical bioequivalence criteria of $80 \%-125 \%$. Atenolol PK is reported to be impacted by fruit juices, most likely due to atenolol being a substrate of transporters such as OATP2B1. ${ }^{37,38}$ Based on the results of this study, apixaban does not modulate transporters for atenolol nor affect its GI absorption. Although coadministration of apixaban and atenolol did result in a small decrease in apixaban exposure (18\% decrease in $\mathrm{C}_{\text {max }}$ and $15 \%$ decrease in $\mathrm{AUC}_{\text {inf }}$ ), the $90 \% \mathrm{CIs}$ for the point estimates fall within the prespecified no-effect criteria. Based on our review of the literature and product label, atenolol does not appear to be a modulator of CYP enzymes or transporters, and has limited potential to be a perpetrator of pharmacokinetic drug interactions. This decrease in apixaban exposure is similar to other factors that impact apixaban exposure, such as body weight, for which subjects $\geq 120 \mathrm{~kg}$ have $23 \%$ lower $\mathrm{AUC}_{\mathrm{inf}}$ and $31 \%$ lower $\mathrm{C}_{\max }{ }^{39}$ This modest decrease in exposure was deemed not clinically relevant, and the dose of apixaban was not adjusted based on body weight alone. ${ }^{2}$ In addition, in the exposure-response prediction for VTE prevention following orthopedic surgery, an 18\% decrease in apixaban exposure resulted in a hazard ratio for bleeding of $0.97 . .^{40}$ Therefore, the decrease in apixaban exposure in the presence of atenolol is not considered clinically meaningful. ${ }^{2}$ The doses used in the atenolol study represent the high end of the recommended dose range for both atenolol (100 mg) and apixaban $(10 \mathrm{mg})$. Thus, it is expected that concomitant administration of apixaban and atenolol at a dose of $100 \mathrm{mg}$ or lower is unlikely to result in a clinically relevant pharmacokinetic drug interaction. The results of these studies indicate that there is no reason to exclude digoxin or atenolol as concomitant medication for patients who are treated with apixaban.

Apixaban is $87 \%$ protein-bound, while atenolol and digoxin are minimally protein-bound ( $\leq 25 \%$ bound). ${ }^{2,19,21}$ These differences in protein-binding properties eliminated concerns about either drug displacing the coadministered drug from protein-binding and affecting unbound levels of coadministered drug. Apixaban has multiple pathways of metabolism and elimination, and does not interfere with the corresponding elimination pathways of atenolol or digoxin.

The single bleeding-related AE of gingival bleeding in the digoxin study does not signal a DDI effect because coadministration of digoxin resulted in an overall decrease in apixaban exposure. Furthermore, digoxin is not associated with a bleeding risk on its own.

\section{Limitations}

These studies did have a few limitations, including a small population composed of healthy subjects and the controlled clinical settings. In the real world, comorbidities and polypharmacy issues would also need to be considered. 


\section{Conclusion}

Apixaban had no effect on the PK of digoxin and there was no clinically relevant interaction between apixaban and atenolol. Based on these results, there are no restrictions on apixaban coadministration with either digoxin or atenolol. Coadministration of digoxin or atenolol with apixaban in healthy subjects was also generally well tolerated.

\section{Acknowledgments}

This study was conducted by Bristol-Myers Squibb and Pfizer Inc. Professional medical writing and editorial assistance was provided by Andy Shepherd and Claire Hall, $\mathrm{PhD}$, at Caudex, funded by Bristol-Myers Squibb and Pfizer Inc.

\section{Author contributions}

Digoxin study: Conception of the work: CF, LSL; design of work: CF, LSL, FL; acquisition of data: ZY, AS, LSL; analysis of data: CF, ZY, LSL, JW; interpretation of data: CF, YS, ZY, LSL, FL; drafting the work: CF, YS, AP, LSL, JW; revising the work critically for important intellectual content: CF, YS, AP, JW, FL; final approval of the version to be published: CF, YS, AP, LSL, JW, FL; agreement to be accountable for all aspects of the work (ensuring that questions related to the accuracy or integrity of any part of the work are appropriately investigated and resolved): CF, YS, AP, LSL, JW. All authors contributed toward data analysis, drafting, and revising the paper, and agree to be accountable for all aspects of the work.

Atenolol study: Conception of the work: CF, ZY, JW; design of work: CF, ZY, JW, FL; acquisition of data: JW, ZY, AS; analysis of data: JW, ZY; interpretation of data: CF, YS, ZY, JW, FL; drafting the work: YS, AP, ZY, JW; revising the work critically for important intellectual content: CF, YS, AP, ZY, JW, FL; final approval of the version to be published: CF, YS, AP, ZY, JW, FL; agreement to be accountable for all aspects of the work (ensuring that questions related to the accuracy or integrity of any part of the work are appropriately investigated and resolved): CF, YS, AP, ZY, JW. All authors contributed toward data analysis, drafting, and revising the paper, and agree to be accountable for all aspects of the work.

\section{Disclosure}

All authors were employees of Bristol-Myers Squibb at the time of research and received salaries and benefits commensurate with employment. The authors report no other conflicts of interest in this work.

\section{References}

1. Bristol-Myers Squibb, Pfizer EEIG. Eliquis ${ }^{\circledR}$ (apixaban tablets) Summary of product characteristics; 2015. Available from: http://www.ema. europa.eu/docs/en_GB/document_library/EPAR_-_Product_Information/human/002148/WC500107728.pdf. Accessed February 25, 2016.

2. Bristol-Myers Squibb Company PI. Eliquis (apixaban) prescribing information; 2015. Available from: http://packageinserts.bms.com/pi/ pi_eliquis.pdf. Accessed February 25, 2016.

3. Connolly SJ, Eikelboom J, Joyner C, et al. Apixaban in patients with atrial fibrillation. N Engl J Med. 2011;364(9):806-817.

4. Granger $\mathrm{CB}$, Alexander JH, McMurray JJ, et al. Apixaban versus warfarin in patients with atrial fibrillation. N Engl J Med. 2011;365(11):981-992.

5. Lassen MR, Raskob GE, Gallus A, Pineo G, Chen D, Portman RJ. Apixaban or enoxaparin for thromboprophylaxis after knee replacement. N Engl J Med. 2009;361(6):594-604.

6. Lassen MR, Raskob GE, Gallus A, Pineo G, Chen D, Hornick P; ADVANCE-2 investigators. Apixaban versus enoxaparin for thromboprophylaxis after knee replacement (ADVANCE-2): a randomised double-blind trial. Lancet. 2010;375(9717):807-815.

7. Lassen MR, Gallus A, Raskob GE, Pineo G, Chen D, Ramirez LM; ADVANCE-3 Investigators. Apixaban versus enoxaparin for thromboprophylaxis after hip replacement. N Engl J Med. 2010; 363(26):2487-2498.

8. Agnelli G, Buller HR, Cohen A, et al. Apixaban for extended treatment of venous thromboembolism. N Engl J Med. 2013;368(8):699-708.

9. Agnelli G, Buller HR, Cohen A, et al. Oral apixaban for the treatment of acute venous thromboembolism. N Engl J Med. 2013;369(9):799-808.

10. Frost C, Nepal S, Wang J, et al. Safety, pharmacokinetics and pharmacodynamics of multiple oral doses of apixaban, a factor Xa inhibitor, in healthy subjects. Br J Clin Pharmacol. 2013;76(5):776-786.

11. Raghavan N, Frost CE, Yu Z, et al. Apixaban metabolism and pharmacokinetics after oral administration to humans. Drug Metab Dispos. 2009;37(1):74-81.

12. Wang L, Zhang D, Raghavan N, et al. In vitro assessment of metabolic drug-drug interaction potential of apixaban through cytochrome P450 phenotyping, inhibition, and induction studies. Drug Metab Dispos. 2010;38(3):448-458.

13. Zhang D, Frost CE, He K, et al. Investigating the enteroenteric recirculation of apixaban, a factor Xa inhibitor: administration of activated charcoal to bile duct-cannulated rats and dogs receiving an intravenous dose and use of drug transporter knockout rats. Drug Metab Dispos. 2013;41(4):906-915.

14. Zhang D, He K, Herbst J, et al. Characterization of efflux transporters involved in distribution and disposition of apixaban. Drug Metab Dispos. 2013;41(4):827-835.

15. Frost CE, Byon W, Song Y, et al. Effect of ketoconazole and diltiazem on the pharmacokinetics of apixaban, an oral direct factor Xa inhibitor. Br J Clin Pharmacol. 2015;79(5):838-846.

16. Vakkalagadda B, Frost C, Byon W, et al. Effect of rifampin on the pharmacokinetics of apixaban, an oral direct inhibitor of factor Xa. Am J Cardiovasc Drugs. 2016;16(2):119-127.

17. Zhang D, He K, Raghavan N, et al. Metabolism, pharmacokinetics and pharmacodynamics of the factor Xa inhibitor apixaban in rabbits. J Thromb Thrombolysis. 2010;29(1):70-80.

18. Gheorghiade M, van Veldhuisen DJ, Colucci WS. Contemporary use of digoxin in the management of cardiovascular disorders. Circulation. 2006;113(21):2556-2564.

19. GlaxoSmithKline. Lanoxin TM (digoxin) tablets: full prescribing information; 2009. Available from: http://www.accessdata.fda.gov/ drugsatfda_docs/label/2010/020405s004lbl.pdf. Accessed November $1,2013$.

20. Food and Drug Administration. Guidance for industry. Drug interaction studies - study design, data analysis and implications for dosing and labeling: Draft guidance; 2012. Available from: http://www.fda. gov/downloads/Drugs/GuidanceComplianceRegulatoryInformation/ Guidances/ucm292362.pdf. Accessed January 15, 2013. 
21. AstraZeneca Pharmaceuticals LP. Tenormin (atenolol) tablets: full prescribing information; 2012. Available from: http://www1.astrazenecaus.com/pi/tenormin.pdf. Accessed November 1, 2013.

22. Parker G, Ene MD, Daneshmend TK, Roberts CJ. Do beta blockers differ in their effects on hepatic microsomal enzymes and liver blood flow? J Clin Pharmacol. 1984;24(11-12):493-499.

23. Augustijns P, Mols R. HPLC with programmed wavelength fluorescence detection for the simultaneous determination of marker compounds of integrity and P-gp functionality in the Caco-2 intestinal absorption model. J Pharm Biomed Anal. 2004;34(5):971-978.

24. Goldman S, Probst P, Selzer A, Cohn K. Inefficacy of "therapeutic" serum levels of digoxin in controlling the ventricular rate in atrial fibrillation. Am J Cardiol. 1975;35(5):651-655.

25. Smith TW, Butler VP Jr, Haber E. Determination of therapeutic and toxic serum digoxin concentrations by radioimmunoassay. $N$ Engl J Med. 1969;281(22):1212-1216.

26. Alexander JH, Becker RC, Bhatt DL, et al. Apixaban, an oral, direct, selective factor $\mathrm{Xa}$ inhibitor, in combination with antiplatelet therapy after acute coronary syndrome: results of the Apixaban for Prevention of Acute Ischemic and Safety Events (APPRAISE) trial. Circulation. 2009;119(22):2877-2885.

27. Botticelli Investigators, Writing Committee, Buller H, Deitchman D, Prins M, Segers A. Efficacy and safety of the oral direct factor Xa inhibitor apixaban for symptomatic deep vein thrombosis. The Botticelli DVT dose-ranging study. J Thromb Haemost. 2008;6(8): 1313-1318.

28. Lassen MR, Davidson BL, Gallus A, Pineo G, Ansell J, Deitchman D. The efficacy and safety of apixaban, an oral, direct factor Xa inhibitor, as thromboprophylaxis in patients following total knee replacement. J Thromb Haemost. 2007;5(12):2368-2375.

29. Ishizaki T, Oyama Y, Suganuma T, Sasaki T, Nakaya H, Shibuya T, Sato T. A dose ranging study of atenolol in hypertension: fall in blood pressure and plasma renin activity, beta-blockade and steady-state pharmacokinetics. Br J Clin Pharmacol. 1983;16(1):17-25.
30. Yamahira N, Frost C, Fukase H, et al. Safety, tolerability, pharmacokinetics, and pharmacodynamics of multiple doses of apixaban in healthy Japanese male subjects. Int J Clin Pharmacol Ther. 2014;52(7):564-573.

31. Pursley J, Shen JX, Schuster A, et al. LC-MS/MS determination of apixaban (BMS-562247) and its major metabolite in human plasma: an application of polarity switching and monolithic HPLC column. Bioanalysis. 2014;6(15):2071-2082.

32. Diletti E, Hauschke D, Steinijans VW. Sample size determination for bioequivalence assessment by means of confidence intervals. Int J Clin Pharmacol Ther Toxicol. 1991;29(1):1-8.

33. Mant T, Fournie P, Ollier C, Donat F, Necciari J. Absence of interaction of fondaparinux sodium with digoxin in healthy volunteers. Clin Pharmacokinet. 2002;41(Suppl 2):39-45.

34. Boffito M, Pozniak A, Kearney BP, Higgs C, Mathias A, Zhong L, Shah J. Lack of pharmacokinetic drug interaction between tenofovir disoproxil fumarate and nelfinavir mesylate. Antimicrob Agents Chemother. 2005;49(10):4386-4389.

35. Peytavin G, Gautran C, Otoul C, et al. Evaluation of pharmacokinetic interaction between cetirizine and ritonavir, an HIV-1 protease inhibitor, in healthy male volunteers. Eur J Clin Pharmacol. 2005;61(4):267-273.

36. Czendlik CH, Sioufi A, Preiswerk G, Howald H. Pharmacokinetic and pharmacodynamic interaction of single doses of valsartan and atenolol. Eur J Clin Pharmacol. 1997;52(6):451-459.

37. Jeon H, Jang IJ, Lee S, et al. Apple juice greatly reduces systemic exposure to atenolol. Br J Clin Pharmacol. 2013;75(1):172-179.

38. Lilja JJ, Raaska K, Neuvonen PJ. Effects of orange juice on the pharmacokinetics of atenolol. Eur J Clin Pharmacol. 2005;61(5-6):337-340.

39. Upreti VV, Wang J, Barrett YC, et al. Effect of extremes of body weight on the pharmacokinetics, pharmacodynamics, safety and tolerability of apixaban in healthy subjects. Br J Clin Pharmacol. 2013;76(6):908-916.

40. Leil TA, Frost C, Wang X, Pfister M, LaCreta F. Model-based exposureresponse analysis of apixaban to quantify bleeding risk in special populations of subjects undergoing orthopedic surgery. CPT Pharmacometrics Syst Pharmacol. 2014;3:e136.
Clinical Pharmacology: Advances and Applications

\section{Publish your work in this journal}

Clinical Pharmacology: Advances and Applications is an international, peer-reviewed, open access journal publishing original research, reports, reviews and commentaries on all areas of drug experience in humans. The manuscript management system is completely online and includes a very quick and fair peer-review system, which is all easy to use.

\section{Dovepress}

Visit http://www.dovepress.com/testimonials.php to read real quotes from published authors. 